\title{
openheart Gradient changes in bioprosthetic valve thrombosis: duration of anticoagulation and strategies to improve detection
}

\author{
Jwan A Naser (10 , ,2, Ioana Petrescu, ${ }^{2,3}$ Filip lonescu, ${ }^{2,3}$ Vuyisile T Nkomo, ${ }^{2}$ \\ Cristina Pislaru, ${ }^{2}$ Hartzell V Schaff, ${ }^{4}$ Patricia A Pellikka, ${ }^{2}$ Heidi M Connolly, ${ }^{2}$ \\ Alexander C Egbe, ${ }^{2}$ Sorin V Pislaru ${ }^{2}$
}

To cite: Naser JA, Petrescu I, Ionescu F, et al. Gradient changes in bioprosthetic valve thrombosis: duration of anticoagulation and strategies to improve detection. Open Heart 2021;8:e001608. doi:10.1136/ openhrt-2021-001608

JAN and IP contributed equally.

Received 28 January 2021 Accepted 14 May 2021

\section{Check for updates}

(c) Author(s) (or their employer(s)) 2021. Re-use permitted under CC BY-NC. No commercial re-use. See rights and permissions. Published by BMJ.

${ }^{1}$ Internal Medicine, Mayo Clinic, Rochester, Minnesota, USA ${ }^{2}$ Cardiovascular Medicine, Mayo Clinic Division of Cardiovascular Diseases, Rochester, Minnesota, USA

${ }^{3}$ Internal Medicine, Beaumont Health System, Royal Oak, Michigan, USA

${ }^{4}$ Cardiovascular Surgery, Mayo Clinic, Rochester, Minnesota, USA

Correspondence to Dr Sorin V Pislaru; pislaru. sorin@mayo.edu

\section{ABSTRACT}

Objective Bioprosthetic valve thrombosis (BPVT) is increasingly recognised as a major cause of prosthetic dysfunction in the first years postimplantation. How early abnormal gradients can be detected prior to diagnosis and how fast they normalise with anticoagulant therapy is unknown. We set forth to (1) evaluate patterns of increase in gradients prior to BPVT diagnosis and (2) characterise time-course of response to anticoagulation.

Methods Patients treated with warfarin for BPVT (1999-2019) with clinically significant reduction of mean gradients $(\geq 25 \%)$ were identified retrospectively. Recovery was defined as gradient decrease $\geq 50 \%$, to postimplantation or to normal-range gradients per position, model and size. Time-to-BPVT (implantationBPVT diagnosis), potential diagnostic delay (first abnormal gradient by position, model and size-BPVT diagnosis) and time-to-recovery (BPVT diagnosis-complete resolution) were recorded.

Results 77 patients were identified; 32 (42\%) aortic (23 surgical-12 porcine, 11 pericardial; 9 transcatheter); 24 (31\%) mitral; 21 (27\%) tricuspid. Median time-toBPVT was 24, 21 and 10 months, respectively. Potential diagnostic delay was median 21 months for aortic, 4 months for mitral, but 0 for tricuspid. Recovery was significantly faster in mitral than aortic (median 2.5 vs 4.8 months, $\mathrm{p}=0.038$ ) and tricuspid (median 5.9 months, $\mathrm{p}=0.025)$ positions. Porcine aortic valves responded faster than pericardial aortic valves (median 2.9 vs 20.3 months, $\mathrm{p}=0.004$ ).

Conclusion Gradients start to increase months before the clinical BPVT diagnosis. Recovery is faster in mitral and surgical aortic porcine valves; a longer warfarin trial seems indicated in tricuspid and surgical aortic pericardial valves.

\section{INTRODUCTION}

Bioprosthetic valve thrombosis (BPVT) is an increasingly recognised cause of bioprosthetic valve dysfunction. ${ }^{1-4}$ We have previously proposed and evaluated performance of echocardiographic criteria for BPVT diagnosis and shown that patients with clinically manifest BPVT may experience accelerated bioprosthetic valve failure despite successful

\section{Key questions}

What is already known about this subject?

- Bioprosthetic valve thrombosis (BPVT) is a known cause of bioprosthetic valve dysfunction that can be treated by anticoagulation but appears to be associated with accelerated bioprosthetic degeneration.

What does this study add?

- Gradients start to increase months before BPVT diagnosis suggesting a knowledge gap and providing an opportunity for improvement.

- Time of response to warfarin depends on valve position and type.

How might this impact on clinical practice?

- Closer surveillance and increased awareness on BPVT can lead to an earlier diagnosis of BPVT. Timely diagnosis and effective therapy may prevent the accelerated bioprosthetic degeneration we observed in previous studies.

- Knowledge about time of response to warfarin allows tailoring initial anticoagulant therapy according to valve position and type. This could reduce the bleeding risk.

anticoagulant therapy ${ }^{1-3}{ }^{5}$; early detection and adequate therapy may be critical in mitigating irreversible bioprosthetic structural changes.

While multiple reports have shown anticoagulation as effective in BPVT, ${ }^{1-35-9}$ to date no study has reported on the patterns of associated gradient changes before and after diagnosis. This information is critical in defining two key elements in management of BPVT: (1) tailoring strategies to improve early detection of BPVT and (2) assess minimal duration of initial anticoagulant therapy in order to minimise bleeding risk. ${ }^{510}$

\section{METHODS}

\section{Study population}

Only patients who previously agreed to include their data in a retrospective research 
study were included. We retrospectively identified adult patients who were treated with warfarin at our institution for possible BPVT between January 1999 and December 2019 and had clinically significant reduction of mean gradients (MGs) ( $\geq 25 \%$ decrease from MG at time of BPVT diagnosis). Target international normalized ratio (INR) was 2.5 (range 2-3). All patients were managed by their local anticoagulation clinics. Baseline demographical and clinical data were extracted from the electronic medical record.

\section{Definitions}

Evolution of MG before clinical BPVT diagnosis was assessed from all echocardiograms between postimplant ('fingerprint' study) and the clinical diagnosis of BPVT. MGs were considered abnormal when above normal range for the specific prosthesis position, model and size. ${ }^{11-16}$ Patients with gradients above range immediately postimplantation were excluded from this analysis.

A response to anticoagulation was considered present when MG decreased $\geq 25 \%$ from peak at diagnosis. Recovery was defined as decrease of MG to postimplantation baseline level, $\geq 50 \%$ from BPVT diagnosis ${ }^{23}$ or to normal range per position, model and size $\mathrm{e}^{1-16}$ as well as resolution of leaflet thickening/impaired mobility. All patients were started on warfarin at the time of suspected BPVT diagnosis and were maintained at least until recovery or to surgical/percutaneous reintervention.

We defined three-time intervals: time to BPVT (interval between implantation and formal clinical diagnosis of BPVT), time of potential diagnostic delay (interval between first abnormal gradient and clinical diagnosis of BPVT) and time to recovery (interval between BPVT diagnosis and first echocardiographic study demonstrating recovery). The time interval between implantation and first echocardiographic follow-up study was documented. In order to assess the impact of increasing BPVT awareness, we performed separate analysis on patients in the first and second decade of the study.

\section{Statistical analysis}

Normal distribution was assessed with the Shapiro-Wilk test. Categorical data were compared using the Pearson $\chi^{2}$ test and results were reported as frequencies and percentages. Continuous variables were summarised as median and IQR. Normally distributed variables were compared using the Student's t-test, and non-normal variables were compared with the Wilcoxon test. Multiple comparison tests were used as appropriate. Survival analysis was performed by the Kaplan-Meier method. When adjustments were done, the fit proportional hazards method was used for categorical variables and standard least squares with full factorial effects method was used for continuous variables. Time to recovery was measured starting at time of BPVT diagnosis. Subgroup analysis was performed in patients with BPVT in the aortic position for effect of valve type on the evolution and resolution of gradients. Statistical significance was accepted for $p$ value $<0.05$. All analyses were performed with JMP Pro software V.14.1.0 (SAS Institute). The data underlying this article will be shared on reasonable request to the corresponding author.

\section{Patient and public involvement}

There was no direct involvement of patients or public in this research.

\section{RESULTS}

Between January 1999 and December 2019, 77 patients (median (IQR) age 73 (59-83) years; $42(55 \%)$ men) had

Table 1 Demographics and echocardiographic characteristics by valve position

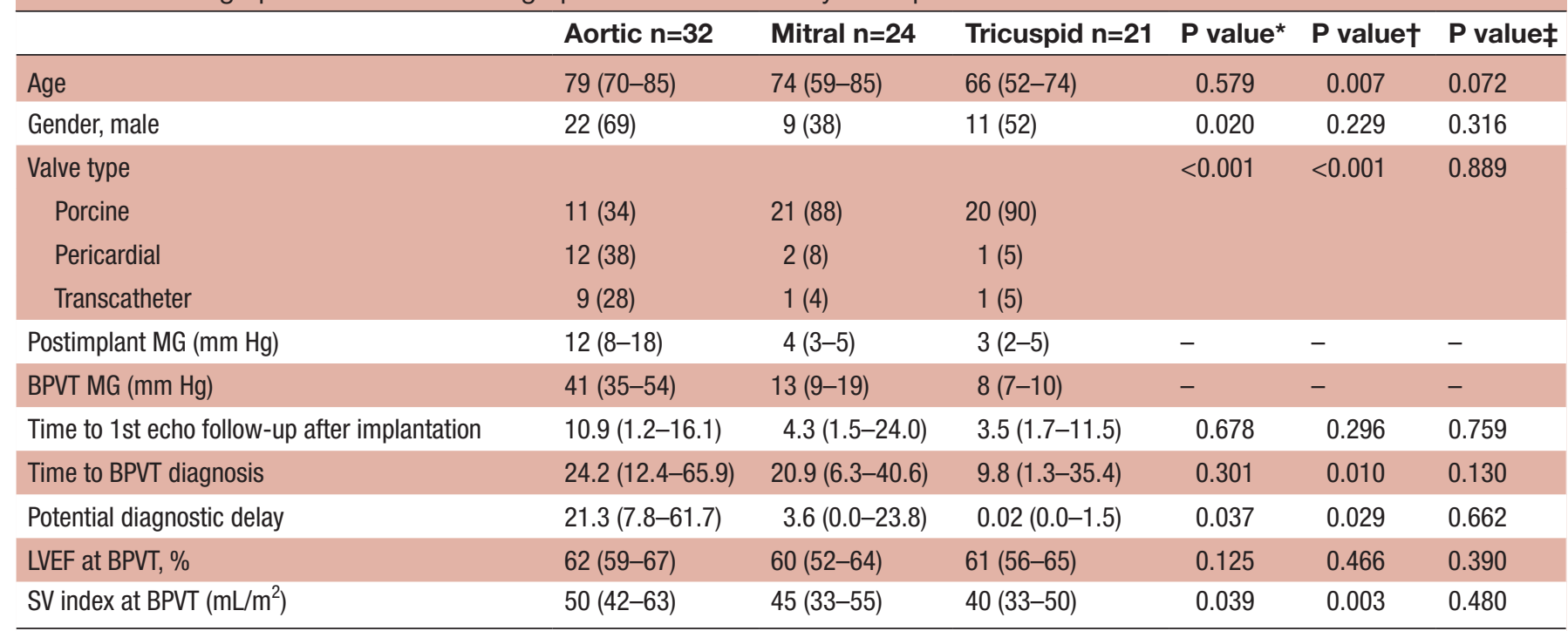

All duration intervals are in months. Numbers are reported as median (IQR) or number (percentage).

${ }^{*}$ Comparison between aortic and mitral positions.

†Comparison between aortic and tricuspid positions.

$\ddagger$ Comparison between mitral and tricuspid position.

BPVT, bioprosthetic valve thrombosis; LVEF, left ventricular ejection fraction; MG, mean gradient; SV, stroke volume. 
$>25 \%$ decrease in MG in response to anticoagulation for presumed BPVT. Of these, $32(42 \%)$ were in the aortic position (surgical, pericardial: 12; surgical, porcine: 11; transcatheter: 9), $24(31 \%)$ in the mitral position and $21(27 \%)$ in the tricuspid position. Demographics and echocardiographic data by valve position are shown in table 1. Some of these patients were included in previous studies. ${ }^{1-35}$ All patients were started on lifelong aspirin after valve implantation. Bar a specific contraindication to anticoagulation, all patients with mitral and tricuspid replacements received 3-month warfarin after the valve implantation. For the aortic position, prior to 2012 patients received only aspirin; after 2012, 3-month warfarin was recommended. At time of BPVT diagnosis, 10 of $77(13 \%)$ patients were hospitalised due to acute heart failure exacerbation.

\section{Time to BPVT and effect of timing of first echocardiographic surveillance}

Median (IQR) time from implantation to BPVT diagnosis was 21 (7-47) months. When analysed by valve position (table 1), time to BPVT in the tricuspid position was shorter than the aortic position $(\mathrm{p}=0.010)$ despite comparable time to first echo follow-up after implantation $(\mathrm{p}=0.296)$. However, comparison of time to BPVT diagnosis between mitral and tricuspid positions did not achieve statistical significance $(\mathrm{p}=0.130)$ although it tended to be shorter in the tricuspid position. Time to first echo follow-up was also comparable $(p=0.759)$ between the mitral and tricuspid positions. A total of 28/77 (36\%) were diagnosed with BPVT within the first year of implantation and $61 / 77(79 \%)$ within 5 years of implantation (figure 1, top panel). Notably, a total of 16/77 (21\%) patients were diagnosed with BPVT more than 5 years after valve implantation.

We noticed a significantly shorter time to first echocardiographic surveillance following transcatheter aortic valve replacement (TAVR) than surgical aortic valve replacement (SAVR) $(1.1(0.8-3.5)$ vs 9.7 (2.3-19.1) months; $\mathrm{p}=0.002)$. Similarly, time to BPVT diagnosis tended to be shorter after TAVR (12.6 (10.529.4) months) than after surgical replacement (SAVR; 37.9 (16.7-69.3) months; $p=0.054$ ). Furthermore, when analysing the relationship between time of first follow-up echocardiographic study and time to BPVT diagnosis,

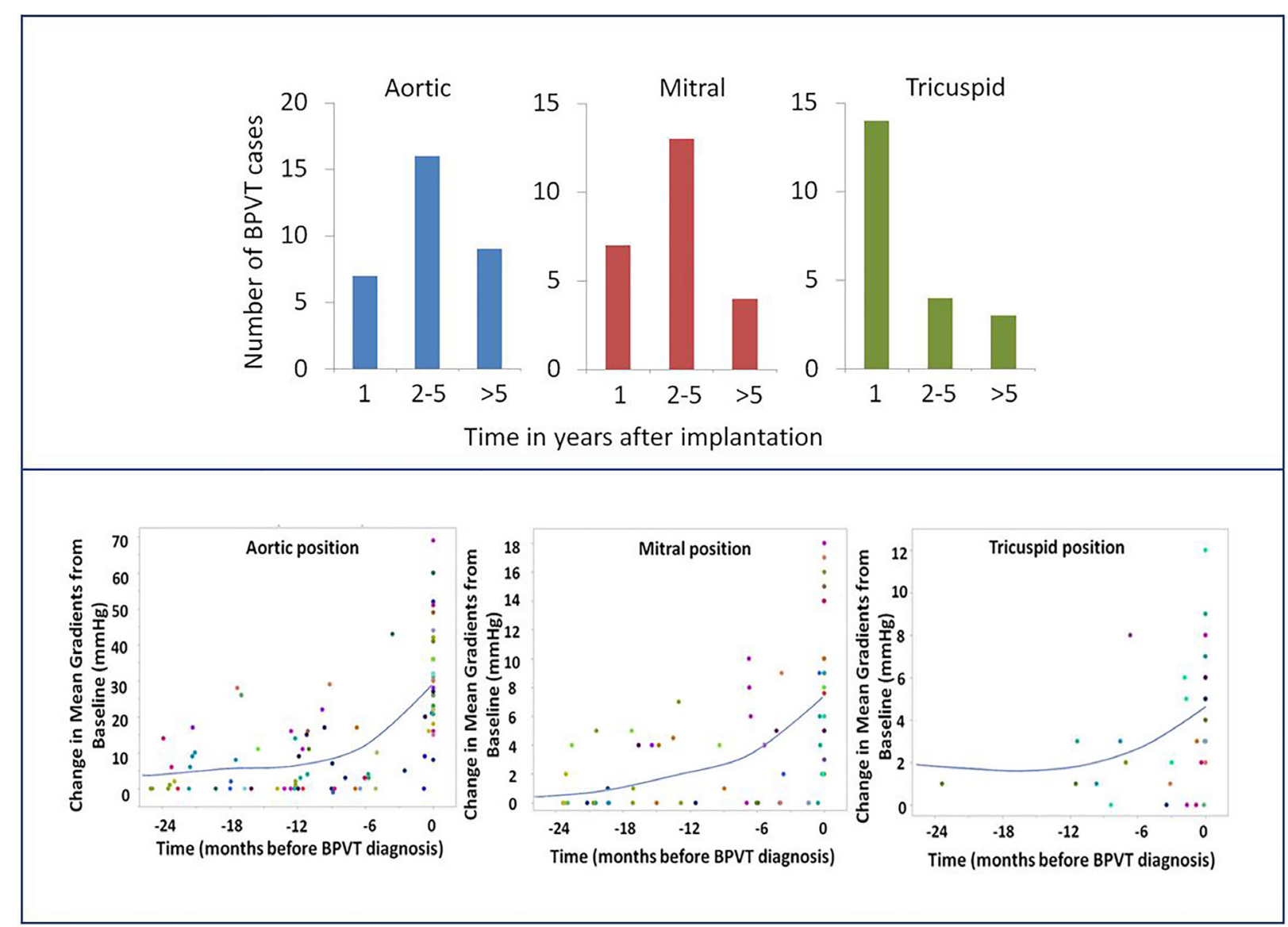

Figure 1 Time to bioprosthetic valve thrombosis (BPVT) and associated potential diagnostic delay. Top panel: Time from valve implantation to the diagnosis of BPVT in the aortic, mitral and tricuspid positions. Most cases are diagnosed in the first 5 years. Bottom panel: Individual patients are coded by colour. The smoother line (cubic spline with lambda 0.05 and standardisedxvalues) shows that gradients start to increase months before the formal diagnosis of BPVT signifying a potential for earlier diagnosis with more strict surveillance and increased awareness. The median number of echocardiographic studies per patient is 3 . 


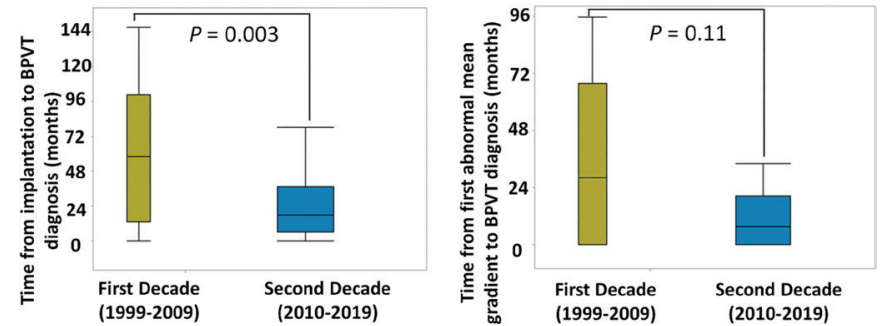

Figure 2 Difference between time to bioprosthetic valve thrombosis (BPVT) diagnosis between the two decades spanning the study duration. Left panel: time to BPVT diagnosis is shorter in the second decade $(n=56)$ than in the first decade $(n=21)$ of the study period. Right panel: time from first abnormal gradient to the formal diagnosis of BPVT is also shorter in the second decade $(n=27)$ than in the first decade $(n=14)$ of the study implicating a shorter diagnostic delay. These findings reflect the role of increased awareness for an earlier diagnosis of BPVT and initiation of appropriate treatment.

there was a moderate-to-strong correlation $(\mathrm{r}=0.7$, $\mathrm{p}<0.001)$. After adjustment for time to first follow-up echo, the difference in time to BPVT diagnosis was no longer significant between TAVR and SAVR patients $(p=0.346)$.

\section{Time of potential diagnostic delay}

Figure 1, bottom panel, shows evolution of MG (as absolute change from postimplant gradients) within the 2 years preceding formal diagnosis of BPVT. The time of potential diagnostic delay was longest for aortic prostheses (21.3 (7.8-61.7) months, $\mathrm{n}=19 ; \mathrm{p}=0.037 \mathrm{vs}$ mitral and 0.029 vs tricuspid). While the delay for mitral (3.6 (0.0-23.8) months, $\mathrm{n}=14$ ) was longer than for tricuspid (0.02 (0.0-1.5) months, $\mathrm{n}=8)$, the difference did not reach statistical significance $(p=0.662)$. There were significant differences between the patterns of diagnosis in the first and second decades of the study period with more patients diagnosed with BPVT (56 in 2010-2019 vs 21 in 1999-2009) at earlier time postimplantation (17.3 (6.636.9) months; $\mathrm{n}=56$ vs 57.7 (13.4-100.6); $\mathrm{n}=21, \mathrm{p}=0.003)$ and with shorter diagnostic delay (7.8 (0.0-20.4) months; $\mathrm{n}=27)$ versus $28.3(0.0-67.7)$ months; $\mathrm{n}=14, \mathrm{p}=0.108$ (figure 2). Of note, there was no significant difference in type of aortic prosthesis (pericardial vs porcine) or in use of $4 \mathrm{D}$ CT scans between the decades $(\mathrm{p}=0.879, \mathrm{p}=0.706$, respectively).

\section{Time to recovery}

A total of 65 patients $(84.4 \%)$ achieved recovery during the study. Of the 12 patients who showed decrease $\geq 25 \%$ of gradients but did not achieve recovery, 2 were lost to follow-up; 5 first responded to warfarin, but had re-escalating gradients within a few years that failed to respond to a second warfarin challenge and required surgical intervention due to pathology-confirmed degeneration; 2 had severe residual symptomatic prosthetic dysfunction despite gradients decreasing $>25 \%$ and underwent early surgery (within 2 months of BPVT diagnosis; pathology confirmed BPVT in both); 1 re-thrombosed immediately due to anti-phospholipid syndrome, and 1 had gradients increase again after initial response due to an unknown mechanism; this last patient has not undergone re- intervention.

Median time (IQR) from BPVT diagnosis to a decrease of $25 \%$ in gradients was $2.7(1.1-5.9)$ months, while median time to recovery was 3.8 (1.4-8.8) months. Age, time of potential diagnostic delay and stroke volume (SV) index had no effect on the rate of resolution of BPVT $(\mathrm{p}=0.491, \mathrm{p}=0.282, \mathrm{p}=0.918$, respectively for recovery). Male gender was only borderline associated with the rate of recovery (HR: 1.66 (1.00-2.76), p=0.051). Early BPVT diagnosis and therapy (shorter time from implantation to clinical BPVT diagnosis and initiation of anticoagulation) was more frequently seen in patients with recovery, but this association did not reach statistical significance (HR $0.93(0.84-1.02)$ per year, $\mathrm{p}=0.13)$. Patients with $>25 \%$ reduction in gradients within first 3 months were highly likely to achieve recovery at long term follow-up (HR of $3.61(2.13-6.11), \mathrm{p}<0.001)$.

\section{Effect of valve position and type on gradient resolution}

While the total numbers are small, we noted a substantial difference in the response to anticoagulation in surgically implanted porcine versus pericardial valves in the aortic position, with a median time to complete resolution of 2.9 and 20.3 months, respectively (figure 3). BPVT in porcine valves was faster to fully recover than in pericardial valves (HR 4.26 (1.46-12.50), p=0.004). Longest duration for recovery was 13.9 months for porcine valves and 26.0 months for pericardial valves. For transcatheter aortic valves, median time to recovery was 3.2 months, and longest time to recovery was 30.7 months. Given the very small number of BPVT in TAVR valves $(\mathrm{N}=9)$, and the fact that BPVT may have been diagnosed at an earlier stage (significantly earlier time of follow-up echocardiography and time to BPVT), we did not include these patients in a direct comparison, as BPVT may have been diagnosed at an earlier stage.

Valve position and type had significant effects on the rates of recovery from BPVT (figure 3). Median time to recovery in the aortic, mitral and tricuspid positions was 4.8, 2.5 and 5.9 months, respectively. Full resolution of BPVT at the mitral position was significantly faster than the aortic (HR 1.91 (1.03-3.54), $\mathrm{p}=0.038)$ and the tricuspid (HR 2.15 (1.09-4.27), $\mathrm{p}=0.025)$ positions. There was no difference between the aortic and the tricuspid positions $(p=0.984)$. Of the patients who achieved recovery, longest duration to recovery in months was 30.7 in the aortic, 5.0 in the mitral and 48.1 in the tricuspid positions.

There was a predominant use of porcine prostheses in mitral (22/24) and tricuspid position (20/21) compared with aortic $(11 / 32)$. When adjusting for valve type, rate of recovery of BPVT was comparable between the aortic and mitral positions $(p=0.226)$. However, mitral BPVT recovery remained faster than the tricuspid $(p=0.034)$. 


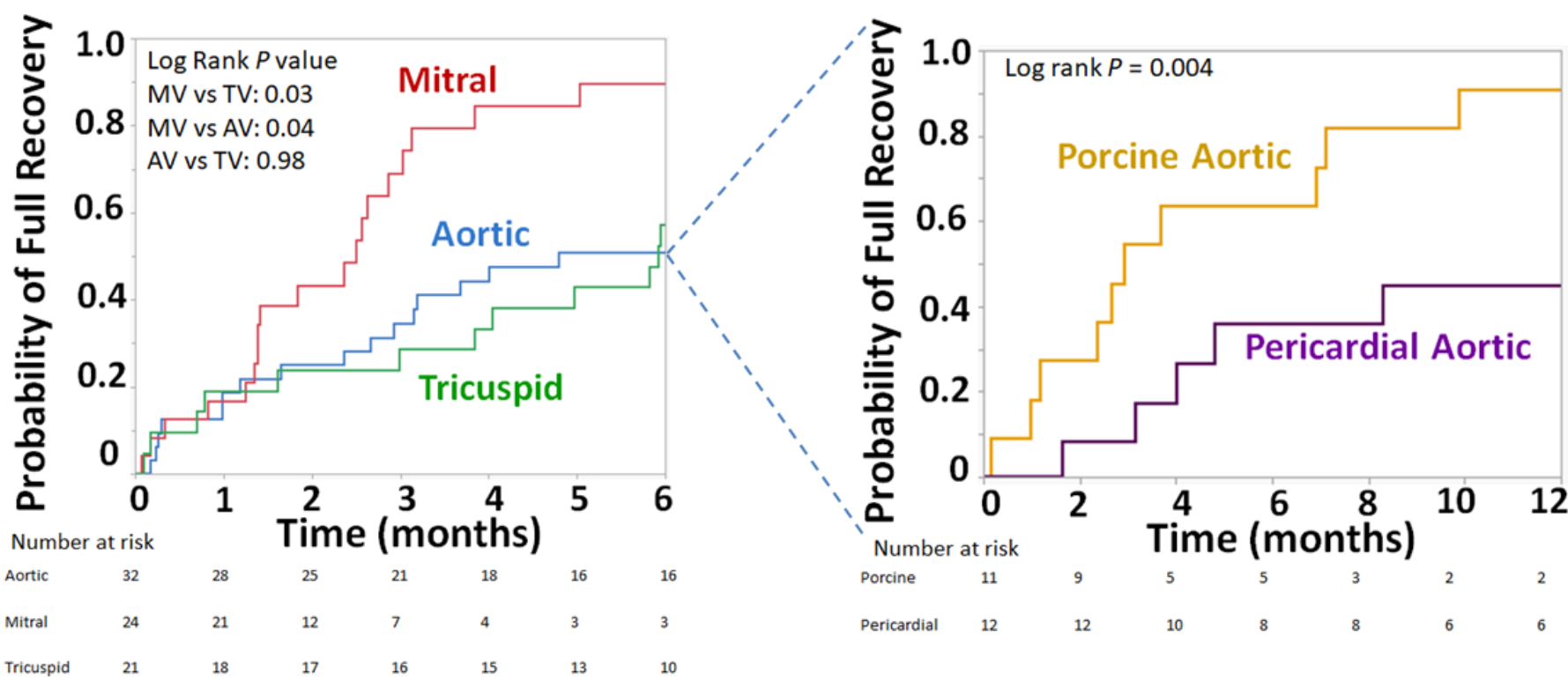

Figure 3 Effect of valve position and type on bioprosthetic valve thrombosis (BPVT) recovery. Left panel: BPVT in the mitral position recovers faster than the aortic and tricuspid positions. Right panel: BPVT in porcine aortic valves recover faster than pericardial aortic valves. AV, aortic valve; MV, mitral valve; TV, tricuspid valve.

\section{DISCUSSION}

To the best of our knowledge, this is the first study that investigates patterns of rise in prosthetic gradients leading to a clinical diagnosis of BPVT and their resolution in response to anticoagulant therapy. Our main findings are: (1) MGs started to increase months before a formal diagnosis of BPVT, suggesting opportunities for improved diagnostic strategies; (2) BPVT recovery tends to be slower in the tricuspid and aortic positions and a warfarin trial should be longer in these cases; (3) surgical pericardial aortic valves seem to recover more slowly than porcine valves; (4) over half of the patients will require anticoagulation for longer than 3 months to achieve recovery.

\section{Diagnostic delays: opportunities for improvement}

MGs started to increase months before the formal diagnosis of BPVT and the initiation of anticoagulation, with median of 21.3 months before aortic BPVT and 6.7 months before mitral BPVT. We believe a contributor was the clinicians' lack of familiarity with BPVT. Indeed, both time from implantation to BPVT diagnosis and time from detection of first abnormal MGs to the formal BPVT diagnosis were shorter in the second decade of the study, most likely due to an increased awareness in the second decade. Moreover, the very short median time from onset of high gradients to diagnosis and treatment in the tricuspid position are in the context of high BPVT awareness in the congenital and carcinoid heart disease groups at our institution (HMC, PAP).

Given our finding of increased valvular gradients significantly before clinically overt BPVT, we hypothesise that a strategy based on early, systematic echocardiographic surveillance may lead to earlier diagnosis of BPVT. Both the 2017 European Society of Cardiology (ESC) and the
2020 American College of Cardiology (ACC)/American Heart Association (AHA) guidelines recommend annual transthoracic echocardiography (TTE) after TAVR. ${ }^{17} 18$ On the other hand, while the 2017 ESC guidelines also recommend annual TTE after surgically implanted valves in asymptomatic patients, the 2020 ACC/AHA guidelines still recommend routine echocardiographic surveillance only at 5 and 10 years postimplantation of surgically implanted valves, then yearly after. ${ }^{19}$ Such an approach would not be able to detect the early subclinical gradient changes we observed in our study, putting patients undergoing surgical valve replacement at a disadvantage when compared with TAVR. The similar 1-year incidence of BPVT noted after SAVR and TAVR in recent clinical trials $^{2021}$ in addition to our observation of no residual difference in time to BPVT between TAVR and SAVR when taking into account time to first follow-up both argue that surveillance should be equally aggressive in SAVR valves.

While increased gradients could be associated with other differential diagnoses (including early prosthetic degeneration, pannus formation, patient-prosthesis mismatch), ${ }^{22}$ recognising that mildly abnormal MGs can be an early sign for subclinical BPVT can set the stage for a closer follow-up that aims for an earlier diagnosis (figure 4). Makkar and colleagues elegantly demonstrated that immobilisation of one leaflet of a prosthetic valve results in only minor increase in MGs towards the upper limit of normal range. ${ }^{4}$ Our data further suggest there is a significant time delay between this initial increase in MG and the overt BPVT picture, providing an opportunity for improvement in current clinical practice. We propose that patients who have imaging features compatible with BPVT diagnosis (abnormal leaflet thickness and motion) 


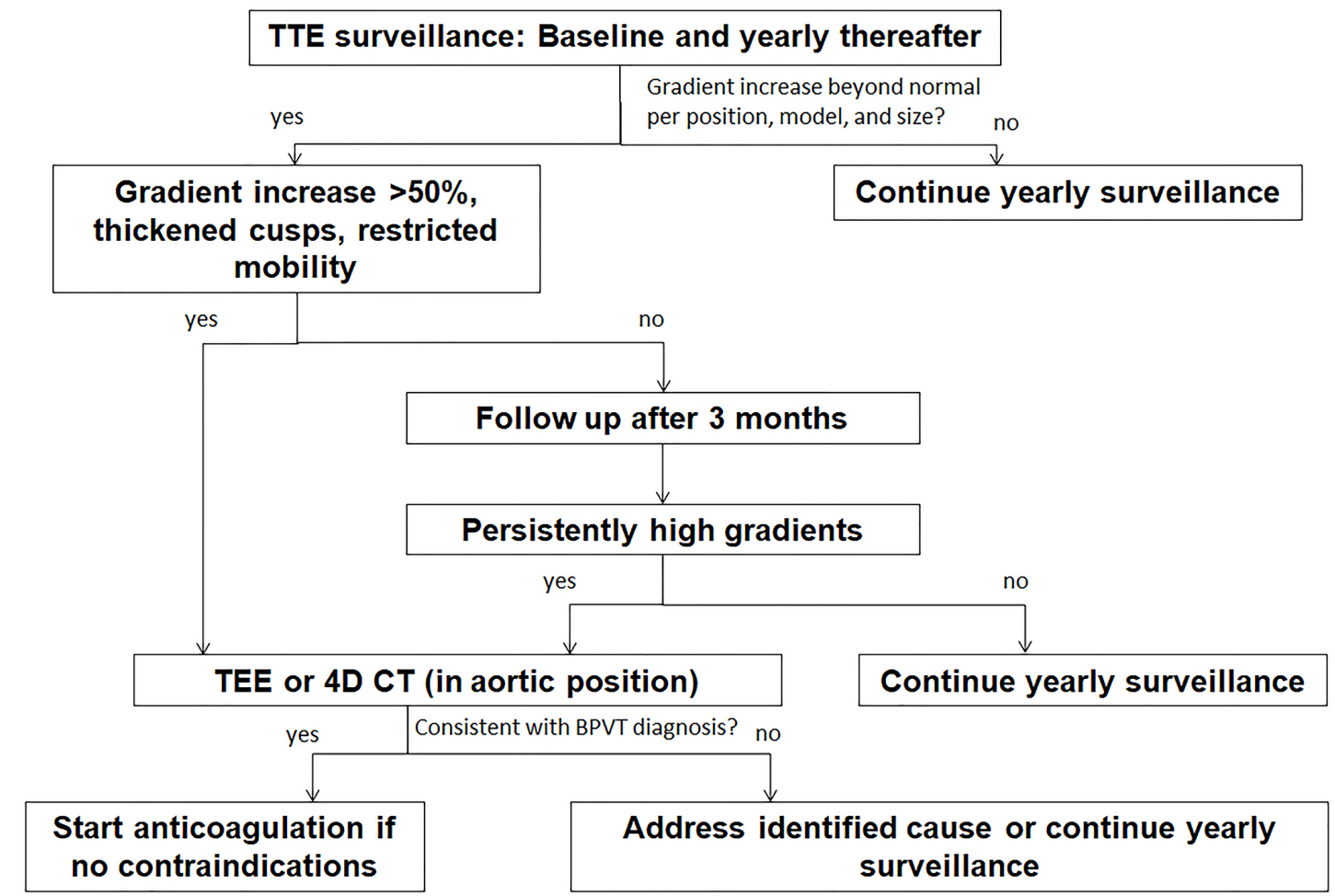

Figure 4 Suggested approach for diagnosis of bioprosthetic valve thrombosis. TEE, transoesophageal echocardiography.

associated with an increase in MG to above normal range should undergo early transoesophageal echocardiography or $4 \mathrm{D}$ CT imaging. ${ }^{42-26}$ If the clinical suspicion of BPVT is low (eg, patient already on anticoagulant therapy; alternative explanation for high gradients; no morphological features of BPVT), we suggest close observation (figure 4). In a small surgical series, we found that atrial fibrillation and hypercoagulable status were associated with BPVT. ${ }^{35}$ In a large TAVR meta-analysis, D'Ascenzo et al have reported other factors associated with BPVT, including larger valve diameter, balloon expandable prostheses, valve in valve procedures, patients with body mass index $>30 \mathrm{~kg} / \mathrm{m}^{2}$ and single antiplatelet therapy. ${ }^{27}$ These patients would benefit from a high index of suspicion and a closer follow-up especially in the setting of increasing gradients.

Cardiac 4D CT has emerged as the imaging modality of choice for aortic bioprosthetic thrombosis. ${ }^{4}$ Presence of hypo-attenuated leaflet thickening (HALT) and restricted leaflet motion are hallmarks of BPVT. ${ }^{4}$ The recent CoreValve Evolut Low Risk and PARTNER 3 trials demonstrated occurrence of BPVT in a significant number of patients at 30 days and 1 year following TAVR or SAVR.$^{20}{ }^{21}$ HALT was not associated with higher gradients measured or with adverse outcomes (deaths, strokes or transient ischaemic attacks (TIAs)) in the CoreValve Evolut Low Risk trial. ${ }^{21}$ On the other hand, the PARTNER 3 trial did show that although the presence of HALT did not significantly affect aortic MG at 30 days or 1 year, patients with HALT at both time points had significantly increased MG at 1 year. ${ }^{20}$ Furthermore, the trial demonstrated higher composite end-point of stroke/TIA and thromboembolic complication rates in patients with HALT. ${ }^{20}$ None of these trials have reported the long-term outcomes. Given our observation of accelerated bioprosthetic failure in overt BVPT $^{20}$ as well as the increased risk of embolic events noted in PARTNER 3, we feel that a strategy designed to diagnose and treat early BPVT could impact long-term outcomes. ${ }^{26}$

\section{Resolution of gradients: duration of initial anticoagulation to observe a full response}

In this retrospective study, median (IQR) time to recovery was 3.8 (1.4-8.8) months. Most likely, true time to recovery is shorter as BPVT may have resolved long before the echocardiogram documenting the response. Furthermore, the observed results are influenced by the highly variable pattern of echocardiographic follow-up ordered by multiple primary cardiologists in an interval spanning two decades. However, important considerations can be inferred from this current study.

First, over half of the patients require $>3$ months of anticoagulation for achieving recovery from BPVT. We suggest that a 3-month interval after initiating anticoagulation for BPVT is the optimal time for first follow-up visit. Indeed, at this stage all patients who responded to anticoagulant therapy already had a decrease in gradients (and can be continued on anticoagulation to full resolution provided the risk of bleeding is acceptable); those who do not respond should be considered for different interventions. Importantly, those patients who experience at least $25 \%$ reduction in gradients within first 3 months have a very high likelihood of recovery; this information can be incorporated in decision-making. 
Second, in terms of valve position, both the aortic and the tricuspid positions seem to need a significantly longer duration of anticoagulation for recovery than does the mitral position. Clinicians must be aware of these differences and prepared to recommend a longer trial of anticoagulation for BPVT in aortic and tricuspid position, especially in the setting of pericardial aortic valves (see below).

Third, in this small cohort, we noted significantly delayed recovery in pericardial aortic valves when compared with porcine valves. Therefore, we suggest that a longer duration of warfarin trial might be needed and should be factored into the discussion with patients diagnosed with BPVT in pericardial valves.

Larger randomised studies are needed to evaluate the role of different anticoagulants including direct oral anticoagulants in treatment of BPVT. Further information is needed whether earlier diagnosis of BPVT can shorten the duration of anticoagulant therapy.

\section{LIMITATIONS}

There are several limitations to our study. First, we defined BPVT according to a combination of echocardiographic findings, response to warfarin, and CT imaging when available, but with no pathology confirmation in most cases. However, echocardiographic criteria have shown excellent sensitivity and specificity in previous studies ${ }^{23}$ and the fact that these patients had both echocardiographic resolution as well as improvement in MGs makes the diagnosis of BPVT reasonable. We only included BPVT cases that were treated with warfarin anticoagulation. Therefore, incidence of BPVT cannot be inferred from this study. Furthermore, given the retrospective nature of the study, we did not have standardised time intervals for follow-up echocardiography after the implantation of valves or after the initiation of warfarin. Hence, reported duration in our study could be an overestimation of the actual time to recovery. It is also important to emphasise here that the time-to-BPVT in our study is the time-toformal BVPT diagnosis which is quite likely an overestimation. Finally, given that patients were followed by their local anticoagulation clinic, we did not have all the INR data to calculate time in therapeutic range.

\section{CONCLUSION}

In conclusion, MGs seems to increase months before the formal diagnosis of BPVT and both closer follow-up and increased awareness could potentially avoid diagnostic delays. Most patients need more than 3 months anticoagulation to achieve recovery. BPVT recovery tends to be slower in the tricuspid position and with pericardial SAVR.

Contributors JAN has drafted the manuscript. All authors have revised it critically for its intellectual content and have agreed to the final version of the text. All authors had substantial contributions to the conception or design of the work; or the acquisition, analysis or interpretation of data for the work. JAN and SVP are responsible for the overall content.
Funding The authors have not declared a specific grant for this research from any funding agency in the public, commercial or not-for-profit sectors.

Competing interests None declared.

Patient consent for publication Not required.

Ethics approval The study was approved by the Institutional Review Board (IRB) and complies with the Declaration of Helsinki.

Provenance and peer review Not commissioned; externally peer reviewed.

Data availability statement Data are available upon reasonable request. Please contact the corresponding author for data requests (Pislaru.Sorin@mayo.edu).

Open access This is an open access article distributed in accordance with the Creative Commons Attribution Non Commercial (CC BY-NC 4.0) license, which permits others to distribute, remix, adapt, build upon this work non-commercially, and license their derivative works on different terms, provided the original work is properly cited, appropriate credit is given, any changes made indicated, and the use is non-commercial. See: http://creativecommons.org/licenses/by-nc/4.0/.

ORCID iD

Jwan A Naser http://orcid.org/0000-0002-6210-7669

\section{REFERENCES}

1 Pislaru SV, Hussain I, Pellikka PA, et al. Misconceptions, diagnostic challenges and treatment opportunities in bioprosthetic valve thrombosis: lessons from a case series. Eur J Cardiothorac Surg 2015;47:725-32.

2 Egbe AC, Pislaru SV, Pellikka PA, et al. Bioprosthetic valve thrombosis versus structural failure: clinical and echocardiographic predictors. J Am Coll Cardiol 2015;66:2285-94.

3 Egbe AC, Connolly HM, Pellikka PA, et al. Outcomes of warfarin therapy for bioprosthetic valve thrombosis of surgically implanted valves: a prospective study. JACC Cardiovasc Interv 2017;10:379-87.

4 Makkar RR, Fontana G, Jilaihawi $\mathrm{H}$, et al. Possible subclinical leaflet thrombosis in bioprosthetic aortic valves. $N$ Engl J Med 2015;373:2015-24.

5 Petrescu I, Egbe AC, lonescu F, et al. Long-Term outcomes of anticoagulation for bioprosthetic valve thrombosis. J Am Coll Cardiol 2020;75:857-66.

6 Butnaru A, Shaheen J, Tzivoni D, et al. Diagnosis and treatment of early bioprosthetic malfunction in the mitral valve position due to thrombus formation. Am J Cardiol 2013;112:1439-44.

7 Egbe A, Pislaru SV, Ali MA, et al. Early Prosthetic Valve Dysfunction Due to Bioprosthetic Valve Thrombosis: The Role of Echocardiography. JACC Cardiovasc Imaging 2018;11:951-8.

8 Jander N, Kienzle R-P, Kayser G, et al. Usefulness of phenprocoumon for the treatment of obstructing thrombus in bioprostheses in the aortic valve position. Am J Cardiol 2012;109:257-62.

9 Oliver JM, Gallego P, Gonzalez A, et al. Bioprosthetic mitral valve thrombosis: clinical profile, transesophageal echocardiographic features, and follow-up after anticoagulant therapy. J Am Soc Echocardiogr 1996;9:691-9.

10 Fitzmaurice DA, Blann AD, Lip GYH. Bleeding risks of antithrombotic therapy. BMJ 2002;325:828-31.

11 Zoghbi WA, Chambers JB, Dumesnil JG, et al. Recommendations for evaluation of prosthetic valves with echocardiography and Doppler ultrasound: a report from the American Society of echocardiography's guidelines and standards Committee and the task force on prosthetic valves, developed in conjunction with the American College of cardiology cardiovascular imaging Committee, cardiac imaging Committee of the American heart association, the European association of echocardiography, a registered branch of the European Society of cardiology, the Japanese Society of echocardiography and the Canadian Society of echocardiography, endorsed by the American College of cardiology Foundation, American heart association, European association of echocardiography, a registered branch of the European Society of cardiology, the Japanese Society of echocardiography, and Canadian Society of echocardiography. J Am Soc Echocardiogr 2009;22:975-1014.

12 Rosenhek R, Binder T, Maurer G, et al. Normal values for Doppler echocardiographic assessment of heart valve prostheses. J Am Soc Echocardiogr 2003;16:1116-27.

13 Külling M, Külling J, Wyss C, et al. Effective orifice area and hemodynamic performance of the transcatheter Edwards Sapien 3 
prosthesis: short-term and 1-year follow-up. Eur Heart J Cardiovasc Imaging 2018;19:23-30.

14 Hahn RT, Leipsic J, Douglas PS, et al. Comprehensive echocardiographic assessment of normal transcatheter valve function. JACC Cardiovasc Imaging 2019;12:25-34.

15 Sagar KB, Wann LS, Paulsen WH, et al. Doppler echocardiographic evaluation of Hancock and Björk-Shiley prosthetic values. J Am Coll Cardiol 1986;7:681-7.

16 Celiento M, Blasi S, De Martino A, et al. The mosaic mitral valve bioprosthesis: a long-term clinical and hemodynamic follow-up. Tex Heart Inst J 2016;43:13-19.

17 Singh M, Sporn ZA, Schaff HV, et al. ACC/AHA versus ESC guidelines on prosthetic heart valve management: JACC guideline comparison. J Am Coll Cardiol 2019;73:1707-18.

18 Baumgartner H, Falk V, Bax JJ, et al. 2017 ESC/EACTS guidelines for the management of valvular heart disease. Eur Heart $J$ 2017;38:2739-91.

19 Otto CM, Nishimura RA, Bonow RO, et al. 2020 ACC/AHA guideline for the management of patients with valvular heart disease: Executive summary: a report of the American College of Cardiology/ American heart association joint Committee on clinical practice guidelines. Circulation 2021;143:e35-71.

20 Makkar RR, Blanke P, Leipsic J, et al. Subclinical Leaflet Thrombosis in Transcatheter and Surgical Bioprosthetic Valves: PARTNER 3 Cardiac Computed Tomography Substudy. J Am Coll Cardiol 2020;75:3003-15.
21 Blanke P, Leipsic JA, Popma JJ, et al. Bioprosthetic Aortic Valve Leaflet Thickening in the Evolut Low Risk Sub-Study. J Am Coll Cardiol 2020;75:2430-42.

22 Pislaru SV, Pellikka PA, Schaff HV, et al. Bioprosthetic valve thrombosis: the eyes will not see what the mind does not know. $J$ Thorac Cardiovasc Surg 2015;149:e86-7.

23 Basra SS, Gopal A, Hebeler KR, et al. Clinical leaflet thrombosis in transcatheter and surgical bioprosthetic aortic valves by four-dimensional computed tomography. Ann Thorac Surg 2018;106:1716-25.

24 Rashid HN, Gooley RP, Nerlekar N, et al. Bioprosthetic aortic valve leaflet thrombosis detected by multidetector computed tomography is associated with adverse cerebrovascular events: a meta-analysis of observational studies. Eurolntervention 2018;13:e1748-55.

25 Holmes DR, Mack MJ. Aortic valve bioprostheses: leaflet immobility and valve thrombosis. Circulation 2017;135:1749-56.

26 Chakravarty T, Søndergaard L, Friedman J, et al. Subclinical leaflet thrombosis in surgical and transcatheter bioprosthetic aortic valves: an observational study. Lancet 2017;389:2383-92.

27 D'Ascenzo F, Salizzoni S, Saglietto A, et al. Incidence, predictors and cerebrovascular consequences of leaflet thrombosis after transcatheter aortic valve implantation: a systematic review and meta-analysis. Eur J Cardiothorac Surg 2019;56:488-94. 